\title{
Editorial
}

\section{Special issue: Energetic electrons in space and laboratory plasmas}

\author{
T. Fülöp ${ }^{1, \dagger}$ and A. P. L. Robinson ${ }^{2}$ \\ ${ }^{1}$ Department of Physics, Chalmers University of Technology, SE-41296 Göteborg, Sweden \\ ${ }^{2}$ Central Laser Facility, STFC Rutherford-Appleton Laboratory, Didcot OX11 0QX, UK
}

(Received 14 March 2016; revised 14 March 2016; accepted 15 March 2016)

Energetic electrons are ubiquitous in plasmas, appearing in, for example, fusion devices, solar flares, laser-produced plasmas, aurorae in planetary magnetospheres, electrical discharges associated with thunderstorms and cosmic rays. Improved understanding of the dynamics of energetic electrons could directly impact the efficiency and beam stability of laser-plasma acceleration, the safety and reliability of next generation magnetic confinement fusion devices, the understanding of lightning initiation and cosmic ray acceleration, as well as improve the diagnostic capabilities for plasmas. Therefore, the subject of energetic electron dynamics has been on the agenda of many research teams all over the world.

One important process leading to suprathermal electrons in plasmas is the so-called runaway mechanism. If the electric field exceeds a critical value, above which the friction force on the charged particles from Coulomb collisions becomes smaller than the force from the electric field, it can detach a fraction of the charged particles from the bulk plasma and accelerate them to relativistic energies. Runaway generation is a candidate mechanism for electron acceleration in solar flares, is frequently observed in electric discharges associated with thunderstorms, and also occurs in magnetic fusion plasmas, when the plasma current is forced to change quickly and a strong parallel electric field is induced. One of the important reasons for understanding runaway electrons is the potential for damage if an uncontrolled runaway electron beam should hit the vessel wall of a fusion device. The knowledge of the dynamics of runaway electrons is absolutely crucial for the development of control methods for these beams.

Energetic electron generation also plays a major role in laser-plasma interaction (LPI). One could even argue that (to use the LPI lingo) 'fast' electron generation underpins most sub-topics in modern laser-plasma physics. Laser-plasma interactions have always been characterised by a significant fraction of the energy being absorbed in suprathermal electrons or electron beams at relativistic intensities. Historically this had an enormous impact on the development of Inertial Confinement Fusion (ICF) as 'hot' electrons were deleterious to the production of highly compressed matter via spherical implosions. In terms of the more modern interests relating to relativistically intense lasers, energetic electrons are a central concern but with a very positive role, as is the case in areas such as wakefield acceleration, fast ignition ICF, ion acceleration, radiation generation, and the generation of warm and hot dense matter.

$\dagger$ Email address for correspondence: tunde@chalmers.se 
In this special issue, we include a range of papers representative of the latest contributions to this rapidly developing field of energetic electrons in plasmas. We gather papers from various branches in plasma physics to illustrate the diversity. The topics range from runaway electrons in fusion plasmas and fast electron transport in laser-produced plasmas, to positron clouds in thunderstorms and energetic electrons in solar flares. 